\title{
Aplicación de las TIC en modelos educativos blended learning: una revisión sistemática de literatura*
}

\section{Application of ICT in blended learning educational models: A systematic review of literature}

\section{Aplicação das TIC em modelos educativos blended learning: uma revisão sistemática da literatura}

\author{
Mayra Alexandra González Aldana \\ Karen Vanesa Perdomo Osorio \\ Yois Pascuas Rengifo
}

Universidad de la Amazonía

Cómo citar: González, M.A., Perdomo, K.V., Pascuas, Y. (2017) Aplicación de las TIC en modelos educativos blended learning: una revisión sistemática de literatura.Sophia 13 (1): 144-154.

\section{Resumen}

En el presente artículo se identifican las tendencias e impacto de la aplicación de las tecnologías de la información y las comunicaciones (TIC) en la modalidad educativa blended learning, también conocido como aprendizaje mezclado, el cual propone hacer una integración entre las clases orientadas en aulas virtuales y las presenciales; dentro de esta investigación se tienen en cuenta resultados y experiencias en distintas áreas educativas haciendo énfasis a la mediación con las TIC. El blended learning, ha logrado gran impacto gracias a la interacción entre el docente y estudiante, generando resultados positivos en el proceso de enseñanza y aprendizaje. Los aspectos metodológicos utilizados con blended learning basados en el uso de las TIC para desarrollar actividades tanto presenciales como virtuales le permite al participante desarrollar habilidades cognitivas, competitivas, pensamiento crítico y constructivo para la resolución de problemas. A través de esta revisión sistemática de literatura, se reconoce la trascendencia del uso de las TIC dentro de esta metodología, ya que permite un proceso dinámico y ajustable del aprendizaje, logrando que este sea pionero en los entornos educativos por su gran eficacia e interacción.

Palabras clave: Aprendizaje virtual, estudiante, formación, modelo, TIC.

*Artículo derivado de la investigación en desarrollo "La gamificación como estrategia ecoalfabetizadora frente a los riesgos de residuos electrónicos en niños de 8 a 10 años de Florencia Caquetá” Grupo de Investigación en Ingeniería de Software de la Universidad de la

Amazonia 
This article identifies the trends and impact of applying information and communication technologies (ICT) in the blended learning educational model, also known as blended learning, which proposes to make an integration between virtual and face-to-face oriented classes in classrooms. In this research, there are taken into account results and experiences in different educational areas, emphasizing mediation with ICT. Blended learning has achieved great impact thanks to the interaction between teachers and students, generating positive results in the teaching and learning process. The methodological aspects used with blended learning based on the use of ICT to develop both online and virtual activities allow the participant to develop cognitive, competitive, critical and constructive thinking skills for problem solving. Through this systematic review of literature, the importance of the use of ICT within this methodology is acknowledged, as it allows a dynamic and adjustable learning process, making it a pioneer in educational environments because of its great effectiveness and interaction.

Key words: Virtual learning, student, training, model, ICT

\section{Resumo}

No presente artigo são identificadas as tendências e o impacto da aplicação das tecnologias de informação e comunicação (TIC) na modalidade educativa blended learning, conhecida também como aprendizagem misturada, que propõe integrar as aulas orientadas em espaços virtuais e as aulas orientadas em espaços presenciais; Nesta pesquisa são tidos em conta resultados e experiências em diferentes áreas educativas, dando especial ênfase à mediação com as TIC. O blended learning, alcançou grande impacto graças à interação entre o professor e o estudante, gerando resultados positivos no processo de ensino e aprendizagem. Os aspectos metodológicos usados com blended learning, baseados no uso das TIC para o desenvolvimento de atividades tanto presenciais como virtuais, permite ao participante desenvolver competências cognitivas, competitivas, pensamento crítico e construtivo para a resolução de problemas. Através desta revisão sistemática da literatura se reconhece a importância do uso das TIC para esta metodologia, uma vez que permite um processo dinâmico e ajustável da aprendizagem, conseguindo que este seja pioneiro nos espaços educativos por sua grande eficácia e interação.

Palavras- chave: aprendizagem virtual, estudante, formação, modelo, TIC.

\section{Introducción}

Los procesos educativos actuales están centrados en ser cada día más eficaces y actualizados, por eso la tendencia en la implementación de las TIC en los procesos de formación, a partir de esta iniciativa surge la necesidad de aplicar nuevos modelos de aprendizaje. En este artículo se plantean los beneficios del modelo educativo blended learning (b-learning), se destaca la aplicabilidad de las herramientas TIC dentro de este proceso, asimismo se realiza un análisis en distintas áreas. Se tuvieron en cuenta diferentes artículos relacionados con blended learning buscados en diferentes bases de datos como Scielo, IEEE, Google académico, Proquest, Science Direct. Posteriormente se realizó una selección de acuerdo con la temática relacionada con blended learning, que intentan responder los siguientes interrogantes: ¿De qué manera ha sido aplicado el blended learning o aprendizaje mezclado?, ¿Cómo han sido implementados los escenarios bajo esta modalidad, en diferentes contextos? Este artículo se centra en un proceso de investigación e indignación sobre experiencias relacionadas con la modalidad de blended learning para realizar un análisis sobre las características, metodologías, conceptualización, importancia y ventajas de la modalidad resaltando la aplicabilidad de las TIC en este modelo educativo. El blended learning permite y promueve el aprendizaje autónomo, autorregular y colaborativo (Ruiz Bolívar, 2008), se reconoce también como aprendizaje mixto, el cual tiene en cuenta sesiones presenciales y otras virtuales, esta práctica conlleva a la interactividad y motivación gracias al trabajo colaborativo de los estudiantes y docentes.

Para desarrollar las actividades se tiene que tener en cuenta tanto un entorno universitario como virtual, se necesita de un espacio de enseñanza semipresencial que permita a los estudiantes expresar sus inquietudes 
acerca de los contenidos establecidos durante el curso (Gámiz Sánchez \& Gallego Arrufat, 2016). Por otro lado, se realiza un análisis a la modalidad de blended learning y se compara con la modalidad de e-learning, aunque se destaca que el aprendizaje mixto no es la perfecta solución a la modalidad de e-learning, se encuentran hallazgos donde el aprendizaje mixto obtiene el $90 \%$ de satisfacción por parte de los estudiantes y docentes de diferentes contextos académicos (Estrada Lizárraga, Zaldívar Colado, Mendoza Zatarain, Nava Pérez, \& García Sánchez, 2013).

\section{Conceptualización}

Esta modalidad es conocida literalmente como el aprendizaje mezclado, por el modo de aprender que combina la enseñanza presencial con la virtualidad y cuya idea clave es la selección de los medios adecuados para cada necesidad educativa (Sanz, Madoz, Gorga, \& González, 2009), (Camacho, Chiappe Laverde, \& López de Mesa, 2012). Se realiza un análisis de la aplicación de las TIC en el modelo de blended learning (Liu \& Zhao, 2010) donde se muestra que por medio de estas herramientas se pueden orientar procesos de aprendizaje a diferentes áreas o campos (BakarNordin \& Alias, 2013) tanto en la educación superior como media, enfocado a las matemáticas, inglés, medicina, ingeniería, pedagogía en el caso de las cuidadoras (niñeras o docentes de niños), (Güzer \& Caner, 2014), (Sánchez Olavarría, 2014), (Cerón Peralta, Gómez Zermeño, \& Abrego Tijerina, 2014). Dentro de las áreas de conocimiento en donde se han aplicado se encuentran las ingenierías, la salud, las matemáticas, biología, sociales, etc.

La mediación con las TIC en la modalidad de blended learning se lleva a cabo en plataformas virtuales, páginas web institucionales, sistemas de administración de aprendizaje (LMS por sus siglas en inglés: Learning Management System) a través de los foros virtuales, correo electrónico, trabajo colaborativo en herramientas en línea, videoconferencias, entre otros (Contreras Bravo, González Guerrero, \& Fuentes López, 2011) (Navas Granados, 2011) (Georgsen \& Lovstad, 2014).

En (Vásquez Lopera \& Arango Vásquez, 2011) se argumenta que la presencia de las TIC en los centros educativos ha generado un aumento notable en la evolución del aprendizaje. El blended learning se constituye en una modalidad educativa emergente, es decir, es un sistema de adaptación (Ramirez, 2008) permitiendo desarrollar habilidades cognitivas, pensamiento crítico y constructivo que van vinculados a la toma de decisiones y la solución de problemas de la cotidianidad (Catalano, 2014).

\section{Ventajas}

La modalidad de blended learning tiene una gran facilidad de apropiación en cuanto a la temática desarrollada durante los procesos de formación, ya que este modelo está ligado con la aplicabilidad de las TIC. Por medio de estas herramientas utilizadas en el proceso de aprendizaje educativo surge en los participantes un gran índice de satisfacción. Es así como (Ruiz Bolívar, 2008) muestra un porcentaje de satisfacción por los estudiantes de un 80,78 en frecuencia relativa, es decir, la aceptabilidad en la implementación del b-learning en las aulas de clase.

El aprendizaje es auténtico e innovador, se centra en el trabajo colaborativo, es decir, el tutor y aprendiz adquieren una gran interacción en cuanto el cumplimiento de dichas tareas durante el curso (Pascal, Comoglio, \& Fernandez, 2012), (Antúnez Sánchez, González Espinosa, Soler Pellicer, Rodríguez Rodríguez, \& Haub, 2014). Logra adecuarse a las necesidades de cada estudiante por ser (aprendizaje autónomo). Adquiere una variedad de recursos para aprender en diversas situaciones y tecnologías. Existe una posible igualdad de oportunidades de aprendizaje, flexibilidad, adaptabilidad, articula las necesidades, orienta al desarrollo de pensamiento crítico y toma de decisiones, por medio de la observación, participación, reflexión, formas socialmente compartidas de conocimiento y pensamiento que le posibilita interactuar con otros, facilitando enriquecer su aprendizaje (Maldonado \& Etcheverry, 2013). Proporciona la comunicación entre el participante $\mathrm{y}$ el cursista permitiendo impartir orientaciones, informaciones y asesorías favoreciendo el intercambio y aprendizaje entre todos (Makhdoom, Khoshhal, Algaidi, Heissam, \& Zolaly, 2013).

Mejora la capacidad en cuanto a la práctica y aplicabilidad del conocimiento visto e integrando una gran experiencia en la interacción y trabajo en equipo, llegando a la apropiacion de sus conocimientos, 
generando gestión y calidad de educación, fortalecimiento en la toma de decisiones, eficacia y efectividad en el rendimiento en los procesos de formación (Slechtova, Vojackova, \& Voracek, 2015).

\section{Características}

El modelo de aprendizaje es virtual dado que está relacionado con los entornos virtuales, de igual manera, el aprendizaje presencial va ligado con las aulas educativas. Incluye modalidad presencial para que los alumnos aclaren dudas y los docentes puedan reforzar más los contenidos programados durante los cursos. Tiene el propósito de ofrecer una mayor flexibilidad en el aprendiz, es decir, se centra en la comodidad educativa donde este pueda aprender de una manera dinámica e influyente obteniendo un resultado positivo. Durante el proceso de aprendizaje se encuentra una separación física entre docente-alumno. En esta modalidad el alumno o aprendiz parte de un aprendizaje independiente.

Como característica principal se tiene que el blended learning es ajustable tanto en los entornos educativos, resaltando el desarrollo de habilidades cognitivas, mejorar comprensión y aplicación de los conocimientos adquiridos, enfrentar problemas del futuro, toma de decisiones, apropiación del manejo de las TIC, flexibilidad horaria, accesibilidad a la información, rapidez en la comunicación, desarrollo y actualización de contenidos.

La modalidad constituye un conjunto de características, dadas por el aprendizaje tradicional como lo son las clases presenciales y el aprendizaje a través las clases virtuales, convirtiéndose en dependientes y complementarias.

\section{Aplicabilidad}

Se ha demostrado en (Ali, Joyes, \& Ellison., 2013) que la modalidad blended learning es el método de enseñanza más apropiado en todos los niveles educativos, ya que este abarca las distintas áreas de los procesos de formación, facilitando a los estudiantes y docentes el entendimiento y comprensión del contenido de los cursos. Se realizan dichos procesos utilizando MOOC (por sus siglas en inglés Masive Open Course Online) (Nakayama, Mutsuura, \& Yamamoto, 2016), el manejo del lenguaje de programación Python, Script, donde los estudiantes hacen énfasis en el pensamiento algorítmico (Rodmunkong, 2015) mediante juegos computacionales (Barik, Everett, Cardona Rivera, Roberts, \& Gehringer, 2013).

La tabla 1 (ver pág sig) nos muestra las diferentes herramientas tecnológicas utilizadas durante las experiencias e investigación de la implementación del blended learning en las aulas educativas, se resalta la relación de los métodos y actividades realizadas para el proceso de aprendizaje-enseñanza.

Esta modalidad da lugar a que los procesos de enseñanza pasen de ser magistrales a ser dinámicos, en este caso actividades presenciales - virtuales motivando al estudiantado a que dicho proceso sea de manera colaborativo bajo la utilización de plataformas en línea como Willow, WebCT (Perez Marin, Santacruz, \& Gómez, 2012), Moodle (Monguet, Fábregas, Delgado, Grimón, \& Herrera, 2006), Edmodo, Mini Blog, videos de presentación, ejercicios en línea (Wai \& Kok Seng, 2014), redes sociales (Yeen-Ju, Mai, \& Selvaretnam, 2015) y juegos en línea, conferencias, tutoriales (Thien Wan, 2015).

La formación teórica como la práctica son evaluadas por diferentes instrumentos, los estudiantes realizan actividades a través de la plataforma Moodle y las clases magistrales, estas últimas por ejemplo se pueden combinar con materiales escritos en soporte electrónico (libros electrónicos o lecturas en archivo PDF o también en espacios virtuales como videos) (Contreras Bravo, González Guerrero, \& Fuentes López, 2011) bajo la aplicación de las TIC, logrando apropiarse de su aprendizaje y no quedarse del avance tecnológico, por último, llegar a adquirir un conocimiento mediante el aprendizaje mixto (Herradón Díez, Blanco Cotano, Pérez Yuste, \& Sánchez Fernández, 2009).

Este modelo también es implementado en la educación superior, se muestra el caso de aplicación con estudiantes de la Facultad de Ingeniería, la interacción a través de una plataforma educativa, de correo electrónico y foros virtuales, en los cuales los estudiantes plasman y resuelven sus inquietudes. Durante la experiencia de una investigación en 
Tabla1. Herramientas implementadas en el modelo educativo blended learning.

\begin{tabular}{|c|c|c|c|c|c|c|c|}
\hline & & & Autores (I & Referencias) & & & \\
\hline \multirow[t]{3}{*}{ Herramientas } & $\begin{array}{l}\text { (Pascal, } \\
\text { Comoglio, \& } \\
\text { Fernandez, } \\
\text { 2012), }\end{array}$ & $\begin{array}{c}\text { (González } \\
\text { Mariño, 2006), }\end{array}$ & $\begin{array}{c}\text { (Ali, Joyes, \& } \\
\text { Ellison, 2014) }\end{array}$ & $\begin{array}{c}\text { (Sivakumar, } \\
\text { Namasivayam, } \\
\text { Al-Atabi, \& } \\
\text { Ramesh, 2013), } \\
\text { (Georgsen \& } \\
\text { Lovstad, 2014), }\end{array}$ & (Lungu, 2013), & $\begin{array}{l}\text { (Makhdoom, } \\
\text { Khoshhal, } \\
\text { Algaidi, } \\
\text { Heissam, \& } \\
\text { Zolaly, 2013), }\end{array}$ & $\begin{array}{c}\text { (Wai \& Kok } \\
\text { Seng, 2014), } \\
\text { (Lau, y otros, } \\
2010), \\
\text { (Dunwell, y } \\
\text { otros, 2011), }\end{array}$ \\
\hline & $\begin{array}{c}\text { (Cerón } \\
\text { Peralta, } \\
\text { Gómez } \\
\text { Zermeño, \& } \\
\text { Abrego } \\
\text { Tijerina, } \\
\text { 2014) } \\
\end{array}$ & $\begin{array}{c}\text { (Vásquez } \\
\text { Lopera \& } \\
\text { Arango } \\
\text { Vásquez, 2011), }\end{array}$ & $\begin{array}{l}\text { (Thien Wan, } \\
\text { 2015), }\end{array}$ & $\begin{array}{l}\text { (Kennepohl, } \\
\text { 2013) }\end{array}$ & $\begin{array}{c}\text { (Yeen-Ju, Mai, } \\
\text { \& Selvaretnam, } \\
\text { 2015), } \\
\text { (Chaiyama, } \\
\text { 2015), }\end{array}$ & $\begin{array}{c}\text { (Nakayama, } \\
\text { Mutsuura, \& } \\
\text { Yamamoto, } \\
2016)\end{array}$ & $\begin{array}{c}\text { (Sorden \& } \\
\text { Ramírez } \\
\text { Romero, 2012) } \\
\text { (Wannapiroon, } \\
\text { 2014) }\end{array}$ \\
\hline & & $\begin{array}{c}\text { (Navas } \\
\text { Granados, } \\
\text { 2011) }\end{array}$ & $\begin{array}{c}\text { (Rajini, Tiwary, } \\
\& \text { Ganapathy, } \\
\text { 2011). }\end{array}$ & & $\begin{array}{c}\text { (Motschnig } \\
\text { Pitrik, 2006) }\end{array}$ & & \\
\hline $\begin{array}{l}\text { Correo } \\
\text { electrónico }\end{array}$ & $\mathrm{x}$ & $\mathrm{x}$ & $\mathrm{x}$ & $\mathrm{x}$ & $\mathrm{x}$ & $\mathrm{x}$ & $\mathrm{x}$ \\
\hline $\begin{array}{l}\text { Plataforma web } \\
\text { institucional }\end{array}$ & $\mathrm{x}$ & & $\mathrm{x}$ & & $\mathrm{x}$ & & \\
\hline Moodle & $\mathrm{x}$ & $\mathrm{x}$ & & & & $\mathrm{x}$ & \\
\hline Foros virtuales & $\mathrm{x}$ & & & & $\mathrm{x}$ & & \\
\hline Redes sociales & $\mathrm{x}$ & $\mathrm{x}$ & $\mathrm{x}$ & $\mathrm{x}$ & & & $\mathrm{x}$ \\
\hline Blogs, mini blogs & & & & $\mathrm{x}$ & $\mathrm{x}$ & & \\
\hline $\begin{array}{l}\text { Encuestas } \\
\text { virtuales } \\
\text { Linea de }\end{array}$ & $\mathrm{x}$ & & & & $\mathrm{x}$ & & \\
\hline $\begin{array}{l}\text { Microsoft } \\
\text { (Word, power }\end{array}$ & $\mathrm{x}$ & $\mathrm{x}$ & & & $\mathrm{x}$ & & $\mathrm{x}$ \\
\hline $\begin{array}{l}\text { point, Excel). } \\
\text { Programas de } \\
\text { lenguaje de }\end{array}$ & & $\mathrm{x}$ & & & & & \\
\hline $\begin{array}{l}\text { programación } \\
\text { (Python, Script, } \\
\text { etc). }\end{array}$ & & & $\mathrm{x}$ & & & & $\mathrm{x}$ \\
\hline Edmodo & & & & $\mathrm{x}$ & & & \\
\hline $\begin{array}{l}\text { Laboratorios } \\
\text { virtuales }\end{array}$ & & & & $\mathrm{x}$ & & & \\
\hline Conferencias & $\mathrm{x}$ & & $\mathrm{x}$ & & & $\mathrm{x}$ & $\mathrm{x}$ \\
\hline Juegos en línea & & & & & & & $\mathrm{x}$ \\
\hline Podcasting & & & & & & $\mathrm{x}$ & $\mathrm{x}$ \\
\hline MOOC & & & & & & $\mathrm{x}$ & \\
\hline
\end{tabular}

Fuente: los autores

el campo de la Física Computacional se utilizó la herramienta computacional eTextBook que apoyó el desarrollo de técnicas (Landau, Paez, \& Bordeianu, 2013). Dentro de los hallazgos se encontró una novedosa tecnología llamada Podcasting aplicada para mejorar la enseñanza y aprendizaje, esta herramienta permite la distribucion de archivos (escuchar, ver - audio, videos) (Thien Wan, 2015).

\section{Evaluación del proceso blended learning}

La modalidad blended learning da lugar a que en la interacción entre docentes y estudiantes, sea necesaria la utilización de entornos virtuales de aprendizaje
(EVA) y diversas herramientas tecnológicas para cumplir con las diferentes secciones de actividades propuestas en los cursos; al momento de realizarse el encuentro presencial los estudiantes tienen la oportunidad de discutir temas específicos tratados virtualmente.

Por otro lado, se realiza una investigación evaluativa "Matriz de desempeño académico" por medio de un curso avanzado de construcción de instrumentos de investigación, aplicada al programa de doctorado del instituto rural (Venezuela), se contó con la participación de 26 graduados y profesores 
universitarios en un rango de edades de 42 años, llevando a cabo clases semi-presencial por medio del cual se obtuvieron los siguientes resultados:

En el desempeño académico se obtuvo que el 95\% del curso memorizó las unidades del contenido realizadas durante el curso, un $90 \%$ considero que se lograron todos los objetivos propuestos en el curso, un $42 \%$ respondió que el nivel de aprendizaje es excelente, un $96.25 \%$ respondió que las expectativas del aprendizaje fueron satisfechas (Krasnova \& Demeshko, 2015). Se aplicaron estrategias de liderazgo, toma de decisiones y relaciones de poder en interacciones mediadas por EVA donde se analiza la relación complementaria o simétrica que se evidenció en las relaciones interpersonales y cómo los estudiantes percibían o asumían el rol de líder en una actividad grupal (Hinojo \& Fernández, 2012), (Vásquez Lopera \& Arango Vásquez, 2011).

Igualmente, (Hinojo, Aznar, \& Cáceres, 2009) de la Universidad Nacional de Lomas de Zamora, destacan la experiencia del blended para determinar el rendimiento académico universitario por medio de entornos virtuales, teniendo en cuenta la percepción del estudiante y el grado de satisfacción respecto de la oferta académica en la modalidad blended learning cuyo resultado es satisfactoria y aceptada. Se trabaja con cursos regulares de carreras de ingeniería que participan de un proyecto de $\mathrm{I}+\mathrm{D}$ que incorpora las TIC. Así mismo, se presenta la experiencia de blended articulada con un LMS (Dunwell, y otros, 2011), donde pretende que los estudiantes desarrollen pensamientos computacionales y de inteligencia artificial mediante el lenguaje de programación Python y Script por medio de ejercicios y realización de videojuegos, permitiéndoles relacionar los conceptos teóricos con la práctica del día a día (Barik, Everett, Cardona Rivera, Roberts, \& Gehringer, 2013).

En el proyecto liderado por (Pascal, Comoglio, \& Fernandez, 2012) se pretendió alcanzar los siguientes resultados: a) Relevar los vestigios digitales en la plataforma para caracterizar las interacciones que tienen lugar entre estudiante- material didáctico y estudiante- docente, b) Identificar, analizar y caracterizar los usos que hacen, estudiantes y docentes, de las herramientas tecnológicas disponibles en el Entorno Virtual de Aprendizaje y c) Describir y comparar el desempeño académico de los alumnos en las distintas aulas virtuales y determinar si existe asociación entre este, la dinámica del uso del EVA, sus creencias y nivel de satisfacción respecto de la oferta educativa y evaluar la existencia de características comunes que permitan establecer alguna tipología. Por medio de los participantes se demostró que en la modalidad de blended learning las estrategias de aprendizaje presencial y virtual se complementan satisfactoriamente, la modalidad presencial durante la estrategia de aprendizaje mezclado favoreció la interacción directa tanto en los participantes como en el docente, además aportó información, aclaración de dudas y retroalimentación, permitiendo la discusión de temas de interés respecto a los objetivos planteados en el curso. Durante el desarrollo de esta modalidad se encontró la necesidad de utilizar: foros, comunicación vía correo con los participantes del curso y profesor, participación en los chats y búsqueda de información en internet.

Las experiencias de aplicación de blended en entornos educativos no va dirigida solo a estudiantes, sino que también se puede implementar para docentes o tutores, se dio a conocer una capacitación a docentes con edades entre 35 y 50 años, los cuales se le dificultaba la utilización de las TIC en los programas de formación dirigidos por ellos, esta experiencia les permitió formarse como docentes idóneos en la aplicabilidad de las TIC en el aula (Catalano, 2014). Dentro del área de inglés también se ha implementado blended Learning por medio de módulos de idiomas que permitieron desarrollar habilidades como es el Listening, Speaking, Writting (Lungu, 2013) (He \& Zhu, 2013).

Se analizó la experiencia de blended en los laboratorios educativos donde se quiere aplicar, estudiar y explorar modos alternativos en el uso de estos (Kennepohl, 2013). En el entorno de pedagogía se encontró que el blended learning es aplicado a docentes de jardines infantiles, los cuales desconocían las herramientas TIC, por ende, desconocían la habilidad de comunicación y enseñanza que promueve su utilización. Se les dio a conocer que la pedagogía va de la mano con la aplicación de las TIC, esto permitió ser más efectivo en relación al rendimiento académico por parte de los niños (Janthon, Songkram, \& Koraneekij, 2015). 


\section{B -learning vs. E- Learning}

Estos modelos de aprendizaje adquieren algunas características similares dentro de ellas están la separación física entre docente y alumnos, incorporación de herramientas TIC, tutorías y aprendizaje independiente. Por medio de esta sección se determina porque el blended learning obtuvo más aceptabilidad en los modelos educativos que el e-learning (Estrada Lizárraga, Zaldívar Colado, Mendoza Zatarain, Nava Pérez, \& García Sánchez, 2013). Primero, se definirá el modelo de aprendizaje e-learning más conocido como el aprendizaje electrónico, este se basa en la educación a distancia por medio de la incorporación de las herramientas TIC (Turpo Gebera, 2009); y como se ha recalcado en este articulo de revisión el concepto de b-learning es el aprendizaje mezclado que incluye herramientas TIC para el mejor desarrollo de los procesos de aprendizaje.

En la modalidad de e-learning se tuvo como resultado un bajo nivel de aprendizaje, debido a que el proceso educativo a distancia, era un proceso tradicional que no abarcaba procesos dinámicos el cual permitía un alto índice de deserción. Se perdía la interacción entre docente y alumno, ya que las tutorías de apoyo a distancia no eran suficientes para aclarar dudas de los aprendices (Antúnez Sánchez, González Espinosa, Soler Pellicer, Rodríguez Rodríguez, \& Haub, 2014). En cuanto a la modalidad de blended learning se obtuvo excelentes resultados, ya que este le permite al estudiante interactuar más entre los compañeros y docentes, debido a que no todas las sesiones son virtuales, se presentan algunas presenciales y se destaca la flexibilidad de horarios centrado en el aprendiz.

\section{Discusión de resultados}

Debido al análisis que se llevó durante el proceso de estudio e investigación de las diferentes experiencias con blended se identificaron los siguientes aspectos, se ha logrado un alto rendimiento académico cuando se ha implementado el modelo de aprendizaje mezclado que esto a su vez permite una gran relación entre estudiante y docente, si se logra una interacción entre estos elementos sin duda alguna se obtendrá una gran comunicación, es decir, un proceso dinámico. Se identifican varios aspectos generales las cuales tienen similitudes no solo con una experiencia de blended sino en diferentes que se caracterizan por tener aspectos y ventajas similares, dentro de los aspectos generales se destaca el blended learning como modelo educativo que permite un alto rendimiento académico en los procesos de formación implementado en las distintas áreas educativas. Le permiten al aprendiz como al docente obtener grandes beneficios en su formación, ya que este modelo está ligado a la aplicabilidad de las TIC y es ajustable a las necesidades educativas. Dentro de la investigación realizada se destacaron los siguientes aspectos:

Tabla 2. Características del modelo educativo blended learning

\begin{tabular}{|c|c|c|c|c|c|c|c|c|c|c|c|}
\hline \multicolumn{12}{|c|}{ Autores (Referencias) } \\
\hline Aspectos generales & $\begin{array}{l}\text { (Pascal, } \\
\text { Comoglio, \& } \\
\text { Ferrandez, } \\
\text { 2012), (Ruiz } \\
\end{array}$ & $\begin{array}{c}\text { (Antúnez } \\
\text { Sinchez, } \\
\text { González } \\
\text { Espinosa, Soler } \\
\end{array}$ & $\begin{array}{l}\text { (Estrada } \\
\text { Lizárraga, } \\
\text { Zaldivar } \\
\text { Colado, } \\
\end{array}$ & $\begin{array}{c}\text { (Camacho, } \\
\text { Chiappe } \\
\text { Laverde, \& } \\
\text { López de Mesa, } \\
\end{array}$ & $\begin{array}{c}\text { (Krasnova \& } \\
\text { Demeshko, } \\
\text { 2015), (Ali, } \\
\text { Joyes, \& } \\
\end{array}$ & $\begin{array}{l}\text { (Hinojo \& } \\
\text { Fernández, } \\
2012), \\
\text { (Gonzzalez } \\
\end{array}$ & $\begin{array}{c}\text { (Cerón Peralta, } \\
\text { Gómez } \\
\text { Zermeño, \& } \\
\text { Abrego } \\
\end{array}$ & $\begin{array}{l}\text { (Ali, Joyes, \& } \\
\text { Ellison, 2014) }\end{array}$ & $\begin{array}{c}\text { (Wai \& Kok } \\
\text { Seng, 2014), } \\
\text { (Rodnunkong, } \\
\text { 2015) } \\
\end{array}$ & $\begin{array}{l}\text { (He \& Zhu, } \\
\text { 2013), (Lungu, } \\
\text { 2013), }\end{array}$ & $\begin{array}{c}\text { (Kennepohl, } \\
\text { 2013), (Hinojo, } \\
\text { Aznar, \& } \\
\text { Cáceres, 2009) } \\
\end{array}$ \\
\hline Alto rendimiento de aprendizaje y enseñanza. & $\mathrm{x}$ & $\mathrm{x}$ & $\mathrm{x}$ & $\mathrm{x}$ & $\mathrm{x}$ & $\mathrm{x}$ & $\mathrm{x}$ & $\mathrm{x}$ & $\mathrm{x}$ & $\mathrm{x}$ & $\mathrm{x}$ \\
\hline $\begin{array}{l}\text { Apropiación de los temas vistos en el proceso } \\
\text { de formación }\end{array}$ & $\mathrm{x}$ & & $\mathrm{x}$ & & $\mathrm{x}$ & $\mathrm{x}$ & $\mathrm{x}$ & & $\mathrm{x}$ & $\mathrm{x}$ & \\
\hline Interacción entre docente y alumno. & & & & $\mathrm{x}$ & & $\mathrm{x}$ & & & & & $\mathrm{x}$ \\
\hline $\begin{array}{l}\text { Permite un proceso de aprendizaje dinámico } \\
\text { y ajustable. }\end{array}$ & $\mathrm{x}$ & $\mathrm{x}$ & $\mathrm{x}$ & $\mathrm{x}$ & & $\mathrm{x}$ & & & $\mathrm{x}$ & & \\
\hline $\begin{array}{l}\text { Desarrollo de habilidades Cognitivas, } \\
\text { pensamiento critico y constructivo. }\end{array}$ & $\mathrm{x}$ & $\mathrm{x}$ & & & & & & $\mathrm{x}$ & & & \\
\hline $\begin{array}{l}\text { Experiencias de Blended realizadas en } \\
\text { educación universitaria. }\end{array}$ & & $\mathrm{x}$ & & & $\mathrm{x}$ & & & $\mathrm{x}$ & & $\mathrm{x}$ & \\
\hline $\begin{array}{l}\text { Fácil comunicación participativa entre los } \\
\text { cursistas y los participantes. }\end{array}$ & & $\mathrm{x}$ & & & & & $\mathrm{x}$ & & & & \\
\hline $\begin{array}{l}\text { Blended realizado en docente del área de } \\
\text { matemáticas (capacitación). }\end{array}$ & & & & $\mathrm{x}$ & & & & & & & \\
\hline $\begin{array}{l}\text { Interfaz destacada por ser organizada, } \\
\text { amigable para el seguimiento de las }\end{array}$ & & & $\mathrm{x}$ & & & & & & & & \\
\hline asignaturas en la modalidad de Blended. & & & & & & & & & & & \\
\hline $\begin{array}{l}\text { Blended Learning basada en el aprendizaje } \\
\text { experiencial de Kolb en el área de salud. }\end{array}$ & & & & $\mathrm{x}$ & & & & & & & \\
\hline $\begin{array}{l}\text { Flexibilidad horaraia, accesibilididad a la } \\
\text { información rapideze na la comunicación, } \\
\text { desarrollo y actualización de contenidos. } \\
\text { Entorno virtual de aprendizaje basado en }\end{array}$ & $\mathrm{x}$ & $\mathrm{x}$ & $\mathrm{x}$ & & $\mathrm{x}$ & & & & & & \\
\hline $\begin{array}{l}\text { Entorno virt ual de aprendinaze basado en } \\
\text { soft ware libre (Moodle) en el proceso de } \\
\text { aprendizaie blended. }\end{array}$ & & $\mathrm{x}$ & & & $\mathrm{x}$ & & & & & & $\mathrm{x}$ \\
\hline $\begin{array}{l}\text { Desarrollo de habilidades como el análisis, } \\
\text { reflexión, capacidad de tomar decisiones. }\end{array}$ & & & & & & & $\mathrm{x}$ & $\mathrm{x}$ & & & \\
\hline Alta implementación de las TIC. & $\mathrm{x}$ & $\mathrm{x}$ & $\mathrm{x}$ & $\mathrm{x}$ & $\mathrm{x}$ & $\mathrm{x}$ & $\mathrm{x}$ & $\mathrm{x}$ & $\mathrm{x}$ & $\mathrm{x}$ & $\mathrm{x}$ \\
\hline $\begin{array}{l}\text { Permite enfrentar problemas del futuro para } \\
\text { solucionarlos. }\end{array}$ & & & & & & & & & $\mathrm{x}$ & & \\
\hline Permite el trabajo en grupo. & & $\mathrm{x}$ & & $\mathrm{x}$ & & & $\mathrm{x}$ & $\mathrm{x}$ & & $\mathrm{x}$ & \\
\hline
\end{tabular}

Fuente: los autores 


\section{Conclusiones}

El blended learning, modalidad de aprendizaje semipresencial que va de la mano con las tecnologías de la información y las comunicaciones, es aplicable a diferentes áreas del conocimiento y permite definir entornos virtuales accesibles con esquemas innovadores.

El análisis de las diversas experiencias educativas acerca de la implementación de la modalidad blended learning, destaca el impacto positivo y ajustabilidad al entorno donde se desarrolle, por estas razones se ha considerado como un gran avance educativo que articula diferentes técnicas, tecnologías y herramientas como las plataformas educativas, foros virtuales, correo electrónico, redes sociales, blogs, mini blogs, conferencias, videojuegos, entre otros.

Los estudiantes o entes participativos también dan su opinión favorable acerca de la modalidad blended learning resaltándola como eficaz y productiva durante su formación; es ajustable para aquellas personas que no cuentan con un horario normal debido a su carga laboral y aporta aspectos claves en los diferentes niveles de formación.

\section{Referencias bibliográficas}

Ali, M. F., Joyes, G., \& Ellison, L. (2014). Building effective small-group team working skill through blended learning at Malaysia Tertiary Institution. Procedia - social and behavioral sciences, 997-1009.

Ali, M. F., Joyes, G., \& Ellison., L. (2013). Using blended learning to enhance students' cognitive presence. Informatics and creative multimedia, 202-208.

Antúnez Sánchez, A. G., González Espinosa, K., Soler Pellicer, Y., Rodríguez Rodríguez, S., \& Haub, J. (2014). Resultados y experencias en la enseñanza de las Matemáticas: La modalidad Blended Learning. Didasc@lia, 5(2), 89-98.

BakarNordin, A., \& Alias, N. (2013). Learning outcomes and student perceptions in using of blended learning in history. Procedia-Social and Behavioral Sciences, 577-585.
Barik, T., Everett, M., Cardona Rivera, R. E., Roberts, D. L., \& Gehringer, E. F. (2013). A community college blended learning classroom experience through artificial intelligence in games. In Frontiers in Education Conference, 1525-1531.

Camacho, J. A., Chiappe Laverde, A., \& López de Mesa, C. (2012). Blended Learning y estilos de aprendizaje en estudiantes universitarios del área de la salud. Educación Médica Superior, 27-44.

Catalano, H. (2014). The opportunity of blended-learning training programs in adult education-ascertaining study. Procedia-Social and Behavioral Sciences, 762-768.

Cerón Peralta, M., Gómez Zermeño, M. G., \& Abrego Tijerina, R. F. (2014). Implementación de B-learning en el nivel superior de educación. Campus virtual , 8-15.

Chaiyama, N. (2015). The development of blended learning management model in developing information literacy skills (BL-ILS Model). International journal of information and education technology, 483-489.

Contreras Bravo, L. E., González Guerrero, K., \& Fuentes López, H. J. (2011). Uso de las TIC y especialmente del blended learning en la enseñanza universitaria. Educación y desarrollo social, 151-160.

Dunwell, I., Petridis, P., Arnab, S., Protopsaltis, A., Hendrix, M., \& de Freitas, S. (2011). Blended game-based learning environments: extending a serious game into a learning content management system. Third international conference on intelligent networking and collaborative systems, 830-835.

Estrada Lizárraga, R., Zaldívar Colado, A., Mendoza Zatarain, R., Nava Pérez, L., \& García Sánchez, O. V. (2013). Percepción de los estudiantes acerca de la implementación de la modalidad educativa blended-learning en la educación superior. Reedies, 1-12. 
Gámiz Sánchez, V., \& Gallego Arrufat, M. J. (2016). Modelo de análisis de metodologías didácticas semipresenciales en educación superior. Educación XX1, 39-61.

Georgsen, M., \& Lovstad, C. V. (2014). Use of blended learning in workplace learning. Procedia-social and behavioral sciences, 774-780.

González Mariño, J. C. (2006). B-learning utilizando software libre, una alternativa viable en educación superior. Complutense de educación, 121-133.

Güzer, B., \& Caner, H. (2014). The past, present and future of blended learning: an in depth analysis of literature. Procedia - social and behavioral sciences , 4596 - 4603.

He, H., \& Zhu, B. (2013). Blended learning of professional english for computer science based on moodle. Fourth international conference on networking and distributed computing, 131-135.

Herradón Díez, R., Blanco Cotano, J., Pérez Yuste, A., \& Sánchez Fernández, J. A. (2009). Experiencias y metodologías "b-learning" para la formación $\mathrm{y}$ evaluación en competencias genéricas en Ingeniería. $L a$ cuestión universitaria, 32-44.

Hinojo, F. J., Aznar, I., \& Cáceres, M. P. (2009). Percepciones del alumnado sobre el blended learning en la universidad. Comunicar, 165-174.

Hinojo, M. ., \& Fernández, A. (2012). El aprendizaje semipresencial o virtual: nueva metodología de aprendizaje en educación superior. Latinoamericana de ciencias sociales, niñez y juventud, 159-167.

Janthon, U, Songkram, N., \& Koraneekij, P. (2015). Work-based blended learning and technological scaffolding system to enhance communication skills for caregivers under local administrative organization, ministry of interior, thailand (Part I). Procedia social and behavioral sciences, 984-991.
Kennepohl, D. K. (2013). Learning from blended chemistry laboratories. Technology for education , 135-138.

Krasnova, T., \& Demeshko, M. (2015). Tutor-mediated support in blended learning. Procedia - social and behavioral sciences, 404-408.

Landau, R. H., Paez, M. J., \& Bordeianu, C. C. (2013). A blended, multimodal access etextbook in computational physics. Computing in science \& engineering, 96-103.

Lau, R. Y., Ip, R. K., Chan, M. T., Kwok, R. C., Wong, S. W., So, J. C., \& Wong, E. Y. (2010). Podcasting: An internet-based social technology for blended learning. Internet Computing, 33-41.

Liu, X., \& Zhao, D. (2010). Research and practice on blended learning model of professional courses in higher-education universities. Second international workshop on education technology and computer science, 415-418.

Lungu, I. (2013). The increasing need for blended-learning models in courses of english for specific courses in Romanian Universities. Procedia - social and behavioral sciences, 470-475.

Makhdoom, N., Khoshhal, K. I., Algaidi, S., Heissam, K., \& Zolaly, M. A. (2013). 'Blended learning' as an effective teaching and learning strategy in clinical medicine: a comparative cross-sectional university-based study. Journal of taibah university medical sciences, 12-17.

Maldonado, C. A., \& Etcheverry, P. (2013). Blended learning 2.0 con mundos virtuales. Ciencia y tecnología, 189-202.

Monguet, J. M., Fábregas, J. J., Delgado, D., Grimón, F., \& Herrera, M. (2006). Efecto del blended learning sobre el rendimiento y la motivación de los estudiantes. Interciencia, 190-196. 
Motschnig Pitrik, R. (2006). Participatory action research in a blended learning course on project management soft skills. Frontiers in Education Conference, 1-6.

Nakayama, M., Mutsuura, K., \& Yamamoto, H. (2016). Lexical analysis of student's learning activities during the giving of instructions for note-taking in a blended learning environment. International journal of information and education technology, $1-6$.

Navas Granados, N. G. (2011). Utilización de un sistema blended learning en el modulo de energías renovables. Enseñanza y divulgación de las ciencias, 171-179.

Pascal, O., Comoglio, M., \& Fernandez, M. (2012). Integración de las TIC en la modalidad blended learning: impacto sobre el rendimiento académico universitario. 913-917.

Pérez Marin, D., Santacruz, L., \& Gómez, M. (2012). A proposal for a blended learning methodology and how to apply it with university students. Procedia - social and behavioral sciences, 5458-5462.

Rajini, R., Tiwary, A., \& Ganapathy, R. (2011). A blended training model to improve learning outcome of it professionals in scripting languages. International conference on technology for education, 285-288.

Ramírez, C. (2008). “La modalidad blended-learning en la educación superior". 1-16.

Rodmunkong, T. (2015). The development of blended learning using internet in computer programming and algorithm. International journal of information and education technology, 442.

Ruíz Bolívar, C. (2008). El blended learning: evaluación de una experiencia de aprendizaje en el nivel de postgrado. Investigación y postgrado, 11-36.
Sánchez Olavarría, C. (2014). B-learning como estrategia para el desarrollo de competencias. El caso de una universidad privada. Rie, $85-100$.

Sanz, C., Madoz, C., Gorga, G., \& González, A. (2009). La importancia de la modalidad "blended learning". Análisis de una experiencia educativa. Te\&et, 47-54.

Sivakumar, S., Namasivayam, S., Al-Atabi, M. T., \& Ramesh, S. (2013). Pre-implementation study of blended learning in an engineering undergraduate programme: taylor's university lakeside campus. Procedia-social and behavioral sciences, 735-743.

Slechtova, P., Vojackova, H., \& Voracek, J. (2015). Blended learning: promising strategic alternative in higher education. Procedia social and behavioral sciences, 1245-1254.

Sorden, S. D., \& Ramírez Romero, J. L. (2012). Collaborative learning, social presence and student satisfaction in a blended learning environment. In advanced learning technologies, 129-133.

Thien Wan, A. (2015). How can learners learn from experience? A case study in blended learning at higher education. International journal of information and education technology, 615-619.

Turpo Gebera, O. (2009). Desarrollo y perspectiva de la modalidad educativa blended learning en las universidades de Iberoamérica. Rie, $1-13$.

Vásquez Lopera, C., \& Arango Vásquez, S. (2011). Propuesta metodológica para la investigación comprensiva: interacciones comunicativas en un entorno virtual de aprendizaje. Lasallista de investigación, 112-123.

Wai, C., \& Kok Seng, E. L. (2014). Exploring the effectiveness and efficiency of blended learning tools in a school of business. Procedia - social and behavioral sciences, 
470-476.

Wannapiroon, P. (2014). Development of research-based blended learning model to enhance graduate students' research competency and critical thinking skills. Procedia - Social and behavioral sciences, 486-490.

Yeen-Ju, H. T., Mai, N., \& Selvaretnam, B. (2015). Enhancing problem-solving skills in an authentic blended learning environment: a Malaysian context. International journal of information and education technology, 841-846. 